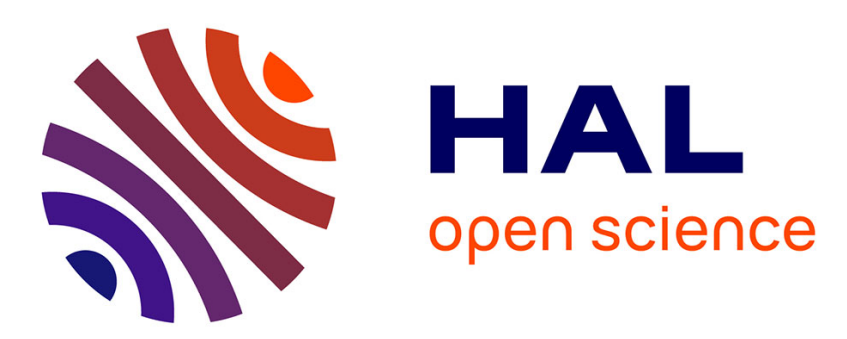

\title{
Integrated optofluidic index sensor based on self-trapped beams in $\mathrm{LiNbO3}$
}

\author{
M. Chauvet, L. Al Fares, B. Guichardaz, F. Devaux, S. Ballandras
}

\section{To cite this version:}

M. Chauvet, L. Al Fares, B. Guichardaz, F. Devaux, S. Ballandras. Integrated optofluidic index sensor based on self-trapped beams in LiNbO3. Applied Physics Letters, 2012, 101 (18), pp.181104. 10.1063/1.4765059 . hal-00776530

\section{HAL Id: hal-00776530 \\ https://hal.science/hal-00776530}

Submitted on 15 May 2021

HAL is a multi-disciplinary open access archive for the deposit and dissemination of scientific research documents, whether they are published or not. The documents may come from teaching and research institutions in France or abroad, or from public or private research centers.
L'archive ouverte pluridisciplinaire HAL, est destinée au dépôt et à la diffusion de documents scientifiques de niveau recherche, publiés ou non, émanant des établissements d'enseignement et de recherche français ou étrangers, des laboratoires publics ou privés. 


\title{
Integrated optofluidic index sensor based on self-trapped beams in $\mathrm{LiNbO}_{3}$
}

\author{
M. Chauvet, L. Al Fares, B. Guichardaz, F. Devaux, and S. Ballandras \\ FEMTO-ST Institute, UMR CNRS 6174, University of Franche-Comté, \\ 16 Route de Gray, 25000 Besançon, France
}

(Received 21 September 2012; accepted 17 October 2012; published online 29 October 2012)

\begin{abstract}
We show that self-trapped beams can form in structured monolithic lithium niobate chips. In particular, they are observed to be unaffected when crossing few hundred microns wide gaps. The technique is employed to fabricate an index sensor constituted of a buried circular optical waveguide crossing a fluidic channel in a lithium niobate substrate. Fluidic channels are realized by precision dicing while the optical waveguides are induced by photorefractive beam self-trapping controlled by the pyroelectric effect. The self-aligning property of this latter waveguides provides a simple fabrication technique of an integrated sensor that accurately measures the refractive index of transparent liquids. (C) 2012 American Institute of Physics. [http://dx.doi.org/10.1063/1.4765059]
\end{abstract}

Association of optical waveguides and microfluidic channels has attracted a lot of attention over the past decade. It indeed offers rapid chemical or biological tests with use of minimum amount of analyte. One important pursued goal is the development of optofluidic lab-on-chip. ${ }^{1,2}$

Such integrated optofluidic devices are based on a variety of substrates. For instance, devices based on glasses, silicon, or polymers ${ }^{3-5}$ have been realized for various applications such as the study of immunoreaction and analysis of fluorescence or spectroscopy. ${ }^{6-8}$ Standard fabrication techniques (photolithography, etching, molding, embossing, etc.) are often employed but the versatility of light writing also offers great solutions. The scanning technique is often used to optically structure material, in particular, to induce waveguides, ${ }^{9}$ but recent studies also reveal that both fluidic channels and waveguides could be laser-written in glass, opening the way to 3-D optofluidic chips. ${ }^{10}$ In the present work, unique properties of self-trapped beams are used to induce integrated waveguides combined with fluidic channels.

Beam self-trapping constitutes a simple fabrication process that can induce low loss buried circular waveguides, in a one step process. Temporary waveguides are potentially interesting for reconfigurable systems ${ }^{11}$ while permanent self-induced waveguides can find applications in self-aligned interconnections ${ }^{12,13}$ or 3-D integrated optics. ${ }^{14,15}$ One of their greatest capabilities is to form waveguides that automatically adapt to the configuration during the writing process. Self-trapped beams thus constitute an efficient tool to form particular waveguides that are sometimes unreachable with other techniques. For instance, tapered waveguides ${ }^{16,17}$ or waveguides with abrupt $90^{\circ}$ turns have been demonstrated. ${ }^{18}$ In this paper, the potential of self-trapped beams is further extended showing that it can form perfectly aligned waveguides crossing fluidic channels. Experiments are realized in lithium niobate $\left(\mathrm{LiNbO}_{3}\right)$ chips where self-trapped beams write waveguides crossing engraved channels. The concept is exploited to realize a compact optofluidic sensor to assess the refractive index of liquids present in the crossed channel.

$\mathrm{LiNbO}_{3}$ samples are cut from a commercial photonic grade z-cut congruent wafer with a precision saw (Disco DAD 321) that provides optical grade dicing. The wafer is maintained by a UV sensitive adhesive film to cut samples of typical size $8 \times 15 \times 0.5 \mathrm{~mm}^{3}$ along $\mathrm{x}, \mathrm{y}$, and $\mathrm{z}$ crystallographic axes, respectively. Polished faces are requested in order to limit losses due to light diffusion. To reach this characteristic, a polymer blade with diamond particles is used. The blade has a thickness of $200 \mu \mathrm{m}$. A constant and high flow of water helps keeping a constant and low temperature for both the blade and the sample during the process. It constitutes a straightforward way to simultaneously dice and polish. Note that this technique was previously used to produce high aspect ratio $\mathrm{LiNbO}_{3}$ ridges with smooth surfaces. ${ }^{19}$ For our study, the same dicing process is used to carve $200 \mu \mathrm{m}$ wide by $200 \mu \mathrm{m}$ deep trenches across the samples. Note that sealing of the trenches that constitute fluidic channels is possible by standard techniques such as thermal bonding of a cover but for the present demonstration trenches were left open.

To induce waveguides in the $\mathrm{LiNbO}_{3}$ chip, visible light coming from either a laser or a laser diode is injected from the side of the sample. Input and output faces of the crystal are observed on a camera via imaging lenses. The overall arrangement is schematically depicted in Figure 1. In this study, beam self-trapping due to the photorefractive effect is controlled by the pyroelectric effect. ${ }^{20}$ To this end, a Peltier element placed under the $\mathrm{LiNbO}_{3}$ sample regulates its temperature with stability better than $0.1^{\circ} \mathrm{C}$.

The principle at the heart of the writing technique consists in injecting a beam inside the $\mathrm{LiNbO}_{3}$ sample that will form its own waveguide as it propagates. Ultimately, the beam is kept confined and propagates with an invariant transverse profile to form a spatial soliton. ${ }^{11,18,20-23}$ The nonlinear optical effect exploited to induce the refractive index change is due to the photorefractive effect. More specifically, a photorefractive spatial soliton can form when electrical charges, generated by light, screen an initial homogeneous electric field present in an electro-optic material. This homogeneous field can be due to an applied voltage as originally shown in $\mathrm{LiNbO}_{3}$ (Refs. 22 and 23) or, as recently discovered, can be induced by a temperature change by pyroelectric effect. ${ }^{20}$ This latter solution greatly simplifies the beam self-trapping control since no electrode is necessary and cautions associated with application of a high voltage are useless. The 


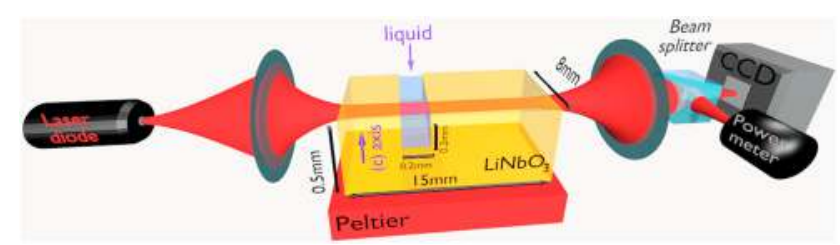

FIG. 1. Schematic diagram of the experimental setup.

electrode free configuration also offers access to novel arrangement such as surface solitons. ${ }^{24}$

A first set of experiments are realized to compare the beam self-trapping behavior in the $\mathrm{LiNbO}_{3}$ sample with or without a channel. Light from a $532 \mathrm{~nm} \mathrm{CW}$ laser is focused to a $12 \mu \mathrm{m}$ full width at half maximum (FWHM) spot (Fig. 2(a)) at the entrance of a sample. The beam is extraordinary polarized and propagates over $15 \mathrm{~mm}$ along the $\mathrm{y}$-axis direction. First, the beam is launched to pass under the fluidic channel. When the sample is at room temperature the beam diffracts to give a Gaussian light distribution of about $130 \mu \mathrm{m}$ FWHM at the exit face of the crystal (Fig. 2(b)). The crystal temperature is then set to $40^{\circ} \mathrm{C}$ at $\mathrm{t}=0$. Self-focusing appears in few minutes for a $60 \mu \mathrm{W}$ light power. Beam confinement and clean-up are obvious after about 2 min (Fig. $2(\mathrm{c})$ ). When a maximum confinement is reached a smooth and efficiently focused spot is observed (Fig. 2(d)) with a $10 \mu \mathrm{m}$ FWHM along z-axis and an $11 \mu \mathrm{m}$ FWHM along $\mathrm{X}$-axis.

Second, the sample is displaced so that the beam crosses the carved channel. In linear regime, the beam is strongly distorted at the exit face (Fig. 2(e)) due to light reflections at the sample top surface and at the bottom of the trench. Notably, Lloyd interferences are clearly seen. However, as selffocusing takes place, beam cleans up and ultimately a confined spot is formed whose size is $8.9 \mu \mathrm{m}$ FWHM along Z-axis and $9.4 \mu \mathrm{m}$ FWHM along X-axis. (Fig. 2(g)). As in the previous experiment, the beam is slightly elliptical. We can conclude that the presence of the trench in the beam path does not perturb beam trapping. Note that, thanks to the self- aligning properties of self-induced waveguides, precise beam launching direction perpendicular to the channel is not required.

We observe that the waveguide induction time is slightly longer when the beam is crossing the channel. Since the photorefractive response time is inversely proportional to the light intensity, this slower response time is consistent with the lower transmitted beam power, due to Fresnel reflections on the trench walls. Note that similar beam trapping characteristics are also obtained for ordinary polarized writing beams (not shown).

After the writing phase, robust waveguides are memorized in the samples. Waveguides lifetime can extend up to months in the dark or when used at low power or at photorefractive insensitive wavelengths. When a self-trapped beam crosses a fluidic channel, two waveguides located on both sides of the channel are present in the medium.

Once beam trapping has occurred, heating is turned off and a probe beam is injected in the photo-induced waveguides. To avoid waveguide degradation, the probe beam optical intensity is reduced on the order of a few $\mathrm{W} / \mathrm{m}^{2}$.

In Figure 3, we present the guided mode for both ordinary and extraordinary polarized probes in waveguides intersecting a fluidic channel. Probe beam wavelength is identical than writing beam $(532 \mathrm{~nm})$ which provides an excellent coupling efficiency better than $90 \%$.

A waveguide written with ordinary polarization guides both ordinary (Fig. 3(a)) and extraordinary (Fig. 3(b)) polarized light. The guided mode with extraordinary polarization is, however, more confined because it is associated with a strong electro-optic coefficient $\left(\mathrm{r}_{33}\right)$, while a lower electrooptic coefficient $\left(\mathrm{r}_{31}\right)$ is associated with ordinary light. For waveguides written with extraordinary light, an efficient waveguide is achieved for an extraordinary polarized probe (Fig. 3(c)) but ordinary light is weakly guided (Fig. 3(d)). These results are in accordance with reported results. ${ }^{20}$

More importantly, these results demonstrate that selftrapped beams form low loss single-mode waveguides that are automatically aligned when crossing gaps of a few
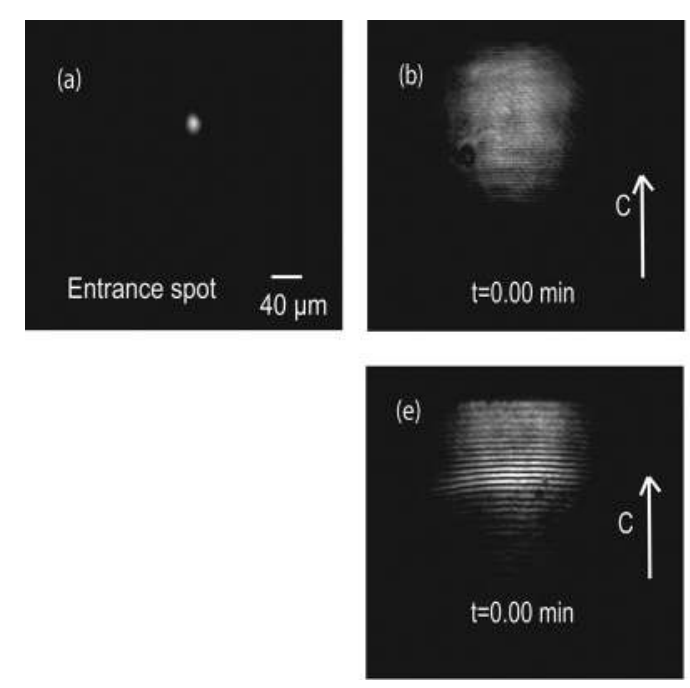
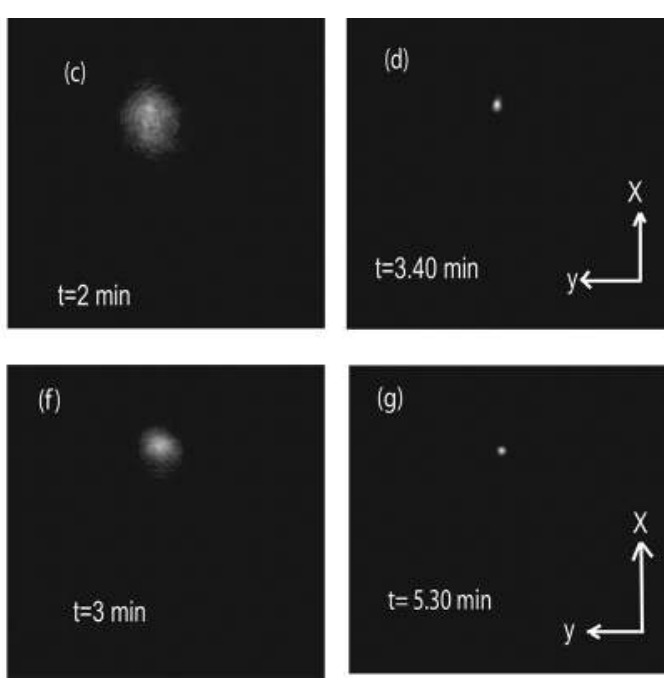

FIG. 2. Beam self-focusing dynamics observed at the exit face of a $15 \mathrm{~mm}$ long $\mathrm{LiNbO}_{3}$ chip when a $12 \mu \mathrm{m}$ FWHM beam is launched in the crystal (a). Dynamics when light beam is passing under the fluidic channel (b)-(d) and when the beam crosses the fluidic channel (e)-(g). Parameters: extraordinary polarization, $\lambda=532 \mathrm{~nm}, \mathrm{p}=60 \mu \mathrm{W}$, crystal temperature: $40^{\circ} \mathrm{C}$. 

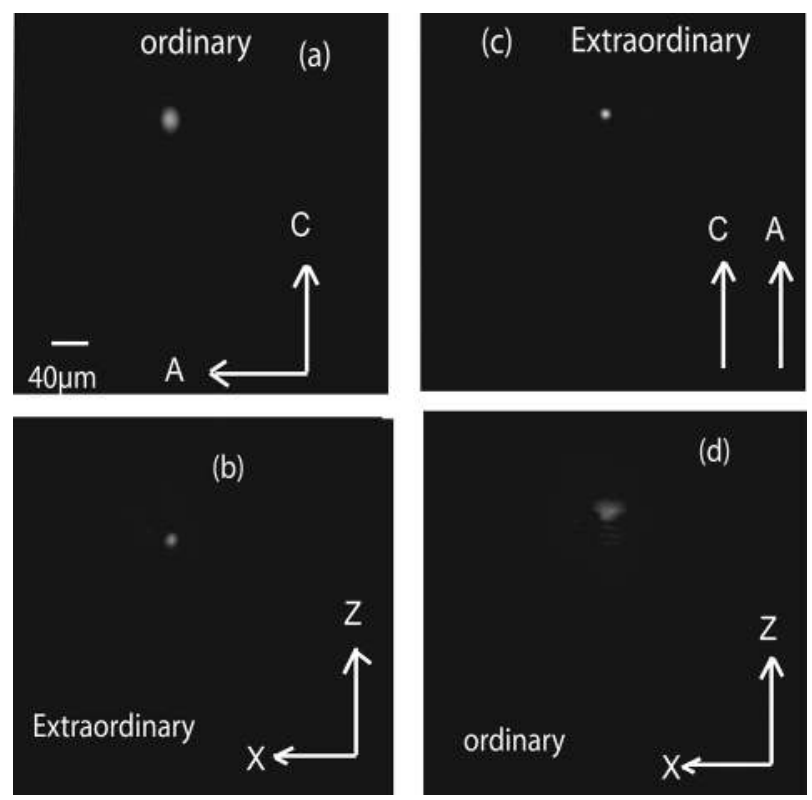

FIG. 3. Images of guided probe beam ordinary or extraordinary polarized at the output of a photo-induced waveguide written with an ordinary (left column) or extraordinary (right column) polarized beam. Writing process parameters: $\mathrm{P}=60 \mu \mathrm{W}, \lambda=532 \mathrm{~nm}$, Temperature: $40^{\circ} \mathrm{C}$. Probe beam parameters: input beam power $<1 \mu \mathrm{W}, \lambda=532 \mathrm{~nm}$, ambient temperature. Arrow A indicates the polarization direction of the writing beam. Arrow $\mathrm{C}$ is the crystal optical axis.

hundred microns wide. The two formed waveguide segments are automatically and perfectly aligned to each other. We would like to underline that additional experiments revealed that formation of perfectly aligned waveguide segments is possible even though the trapped beam is incident at a large angle on the channel.

An optofluidic refractive index sensor is then designed based on the combination of fluidic channels and buried light induced waveguides integrated on a monolithic $\mathrm{LiNbO}_{3}$ substrate. Miniaturized refractive index sensors are useful to accurately and rapidly analyze various phenomena such as variation of composition or kinetic reactions. ${ }^{25,26}$

To fabricate the refractive index sensor, a waveguide crossing perpendicularly a $200 \mu \mathrm{m}$ wide fluidic channel is induced by beam self-trapping. The configuration is similar than in Figure 1 except that the light source is a diode laser at the central wavelength of $640 \mathrm{~nm}$ coupled to a fibered single-mode polarization maintaining fiber. The fiber is positioned to form a $12 \mu \mathrm{m}$ (FWHM) spot size at the entrance face of the $\mathrm{LiNbO}_{3}$ chip. This compact light source operates both for the writing stage and for the subsequent characterization of the index sensor.

For the waveguide writing process, the diode current is set above the threshold current to give a quasimonochromatic light of about $300 \mu \mathrm{W}$. The light beam is extraordinary polarized. The $\mathrm{LiNbO}_{3}$ chip is then heated to $40{ }^{\circ} \mathrm{C}$ to trigger the self-focusing effect. A waveguide crossing the channel is formed in less than $5 \mathrm{~min}$.

To test the realized device, a probe beam is launched in the waveguide and the output power is monitored while a fluid is inserted in the channel. The very same light source arrangement than for the written phase is used which guarantees a very good coupling in the waveguide. For this test phase, the current of the laser diode is set below the threshold current to provide optimal conditions. First, it gives a weak light power, which guarantees that the waveguide will not decay. Second, the emitted spectrum is wide enough to be considered as an incoherent source to avoid Fabry-Perot resonances. Indeed the $10 \mathrm{~nm}$ wide spectrum is more than one order of magnitude larger than the free spectral range of the Fabry-Perot cavity formed by the fluidic channel walls. The sensor light transmittance is monitored with a power meter while the fluid is inserted in the fluidic channel.

Figure 4 presents the observed transmitted light evolution for a typical measurement. The transmitted light is normalized to $T_{0}$, the transmission of an empty channel. The initially empty channel is filled with a droplet of ethanol at $\mathrm{t}=14 \mathrm{~s}$. The transmission increases to a value 1.230. This transmission change is defined as $\Delta T={ }^{T} / T_{0}$, where $\mathrm{T}$ is the sensor transmission filled with the liquid to analyze. The chip temperature is intentionally left at $40{ }^{\circ} \mathrm{C}$ so that the ethanol evaporates from the open channel. At $t=24 \mathrm{~s}$, the meniscus present at the surface of the liquid reaches a similar position than the light beam crossing the channel. The transmittance consequently decays dramatically due to light reflection at the meniscus surface. From $\mathrm{t}=25 \mathrm{~s}$ to about $29 \mathrm{~s}$, light is totally reflected by the meniscus. Finally, when no more liquid is present in front of the waveguide the initial transmission $T_{0}$ is recovered ( $\mathrm{t}>30 \mathrm{~s}$ ).

The liquid can affect light transmission due to several factors such as absorption, diffusion, or reflection. In our case, $\Delta T$ is dictated by the liquid refractive index since we consider homogeneous transparent liquids.

To calculate $\Delta T$, multiple Fresnel reflections on both walls of the channel are first taken into account. For our configuration, the liquid of refractive index $n_{L}$ is embedded in $\mathrm{LiNbO}_{3}$ of refractive index $n_{1}$. The transmission coefficient of this cavity crossed at normal incidence with a large spectrum (low coherence) light beam is given by

$$
T_{F}=\frac{2 n_{1} n_{L}}{n_{1}^{2}+n_{L}^{2}} .
$$

In Figure 5, the dashed curve represents the calculated $\Delta T$ due to only Fresnel reflection versus the liquid refractive index $n_{L}$. The refractive index $\mathrm{n}_{1}$ of $\mathrm{LiNbO}_{3}$ at the wavelength of $640 \mathrm{~nm}$ is taken from Ref. 27. Note that since the refractive index of $\mathrm{LiNbO}_{3}$ is large $(\approx 2.2) \Delta T$ varies

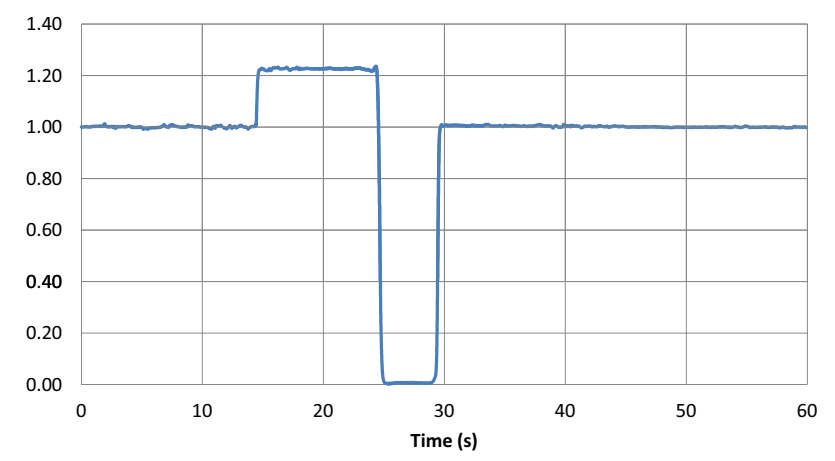

FIG. 4. Measured evolution of the sensor optical normalized transmission with ethanol. 


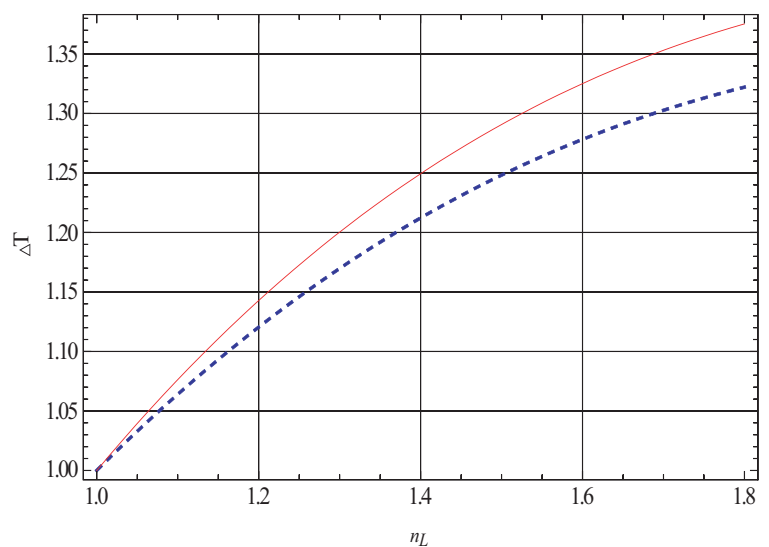

FIG. 5. Variation of the normalized transmission $\Delta T$ as a function of the refractive index $n_{L}$ of the fluid present in the channel. Dashed curve: influence of Fresnel reflection Solid curve: influence of Fresnel reflection and diffraction.

significantly when liquids are analyzed. The refractive index of ethanol at the sensing wavelength being 1.360 , the predicted $\Delta T$ value due to Fresnel reflections is 1.195 which is weaker than the experimental value of 1.230 in Figure 4. This offset is attributed to the influence of beam free diffraction inside the channel. In the gap, light diffracts before reaching the opposite waveguide. As a consequence, coupling efficiency $T_{C}$ due to beam and guided mode overlap is altered. The self-trapping technique implies that waveguides on both side of the channel are perfectly aligned and possess the same guiding characteristics. With the hypothesis of Gaussian beam profiles, $T_{C}$ is given by ${ }^{28}$

$$
T_{C}=\frac{2 W_{1} W_{2}}{W_{1}^{2}+W_{2}^{2}}
$$

where $W_{1}$ is the waist of the guided beam and $W_{2}$ is the waist of the beam after travelling across the gap of width $\mathrm{L}$. According to Gaussian beam propagation theory, $W_{2}$ is given by

$$
W_{2}=W_{1} \sqrt{1+\left(\frac{\lambda L}{\pi n_{L} W_{1}^{2}}\right)^{2}} .
$$

The total transmission coefficient of the device becomes $T=T_{F} T_{C}$. The solid curve in Fig. 5 presents the corresponding $\Delta T$ variation for an $8.1 \mu \mathrm{m} \mathrm{FWHM}\left(W_{1}=6.8 \mu \mathrm{m}\right)$ guided mode. This mode size gives a theoretical value for $\Delta T$ of 1.230 which is identical to the one observed in the experiment with ethanol $\left(n_{L}=1.360\right)$. Moreover, a similar mode size is observed experimentally in Figure 3. We can, thus, conclude that the solid curve from Figure 5 is a valid calibration curve for the realized index sensor.

Refractive index of methanol is then tested with the sensor. The measurement gives a refractive index of 1.317 at $640 \mathrm{~nm}$, which is in fair agreement with the reported value of 1.320. The refractive index sensor sensitivity is about $5 \times 10^{-3}$ for index between 1 and 1.8 . The sensitivity can be improved by using a more elaborated detection scheme.
In conclusion, we have shown that self-trapped beams represent a straightforward technique to fabricate optofluidic components. Specifically, low loss light-induced waveguides crossing fluidic channels have been formed in a monolithic $\mathrm{LiNbO}_{3}$ chip. Fluidic channels have been realized by optical grade sawing, while buried optical waveguides have been induced by photorefractive beam self-trapping. A miniature sensor has been designed and fabricated to measure index of refraction of transparent liquids with a precision of $5 \times 10^{-3}$. Above all, this work demonstrates the potential of beam selftrapping to easily fabricate devices that are composed of precisely aligned waveguides crossing interfaces. This fabrication technique could be applied to other materials such as photosensitive polymer.

Financial support by Agence National de la Recherche for project CHARADES (ANR MatePro 2012) is gratefully acknowledged.

${ }^{1}$ D. Psaltis, S. R. Quake, and C. Yang, Nature 442, 381-386 (2006).

${ }^{2}$ C. Monat, P. Domachuk, and B. J. Eggleton, Nat. Photonics 1, 106-114 (2007).

${ }^{3}$ V. Lien, Y. Berdichevsky, and Y. H. Lo, IEEE Photonic Technol. Lett. 16, 1525-1527 (2004).

${ }^{4}$ J. M. Ruano, A. Glidle, A. Cleary, A. Walmsley, J. S. Aitchison, and J. M. Cooper, Biosens. Bioelectron. 18, 175-184 (2003).

${ }^{5}$ J. Cooper McDonald and G. M. Whitesides, Acc. Chem. Res. 35, 491-499 (2002).

${ }^{6}$ A. Ymeti, J. S. Kanger, J. Greve, G. A. J. Besselink, P. V. Lambeck, R. Wijn, and R. G. Heideman, Biosens. Bioelectron. 20, 1417-1421 (2005).

${ }^{7}$ Y. Tunga, M. Zhanga, C. T. Linb, K. Kurabayashia, and S. J. Skerlos, Sens. Actuators B 98, 356-367 (2004).

${ }^{8}$ L. Malic and A. G. Kirk, Sens. Actuators, A 135, 515-524 (2007).

${ }^{9}$ D. Homoelle, S. Wielandy, and A. L. Gaeta, Opt. Lett. 24, 1311-1313 (1999).

${ }^{10}$ R. Osellame, V. Maselli, R. Martinez Vazquez, R. Ramponi, and G. Cerullo, Appl. Phys. Lett. 90, 231118-231120 (2007).

${ }^{11}$ L. Friedrich, G. I. Stegeman, P. Millar, C. J. Hamilton, and J. S. Aitchison, Opt. Lett. 23, 1438-1440 (1998).

${ }^{12}$ T. Yamashita, M. Kagami, and H. Ito, J. Lightwave Technol. 20, 1556 (2002).

${ }^{13}$ K. Dorkenoo, O. Crégut, L. Mager, F. Gillot, C. Carre, and A. Fort, Opt. Lett. 27(20), 1782-1784 (2002).

${ }^{14}$ V. Coda, M. Chauvet, F. Pettazzi, and E. Fazio, Electron. Lett. 42, 463465 (2006).

${ }^{15}$ A. S. Kewitsch and A. Yariv, Opt. Lett. 21, 24-26 (1996).

${ }^{16} \mathrm{~S}$. J. Frisken, Opt. Lett. 18, 1035-1037 (1993).

${ }^{17}$ L. Al Fares, F. Devaux, and M. Chauvet, Opt. Lett. 36, 3843-3845 (2011).

${ }^{18}$ R. Jäger, S. P. Gorza, C. Cambournac, M. Haelterman, and M. Chauvet, Appl. Phys. Lett. 88, 061117 (2006).

${ }^{19}$ N. Courjal, B. Guichardaz, G. Ulliac, J. Y. Rauch, B. Sadani, H. H. Lu, and M. P. Bernal, J. Phys. D: Appl. Phys. 44, 305101 (2011).

${ }^{20}$ J. Safioui, F. Devaux, and M. Chauvet, Opt. Express 17, 22209 (2009).

${ }^{21}$ M. Segev, G. C. Valley, B. Crosignani, P. Di Porto, and A. Yariv, Phys. Rev. Lett. 73, 3211-3214 (1994).

${ }^{22}$ E. Fazio, F. Renzi, R. Rinaldi, M. Bertolotti, M. Chauvet, W. Ramadan, A. Petris, and V. I. Vlad, Appl. Phys. Lett. 85(12), 2193-2195 (2004).

${ }^{23}$ E. Fazio, W. Ramadan, A. Petris, M. Chauvet, A. Bosco, V. I. Vlad, and M. Bertolotti, Appl. Surf. Sci. 248(1), 97-102 (2005).

${ }^{24}$ J. Safioui, E. Fazio, F. Devaux, and M. Chauvet, Opt. Lett. 35(8), 12541256 (2010).

${ }^{25}$ W. Yuan, G. E. Town, and O. Bang, IEEE Sens. J. 10(7), 1192-1199 (2010).

${ }^{26}$ T. Mappes, J. Mohr, K. Mandisloh, M. Schelb, and B. Ross, Proc. SPIE 6992, 69920L (2008).

${ }^{27}$ U. Schlarb and K. Betzler, Phys. Rev. 48, 15613 (1993).

${ }^{28}$ P. D. Bear, Appl. Opt. 19, 2906-2909 (1980). 